\title{
MARITIME ANOMALY DETECTION IN FERRY TRACKS
}

\author{
C.Zor, J.Kittler \\ Centre for Vision, Speech and Signal Processing (CVSSP) \\ University of Surrey, United Kingdom, GU2 7XH
}

\begin{abstract}
This paper proposes a methodology for the automatic detection of anomalous shipping tracks traced by ferries. The approach comprises a set of models as a basis for outlier detection: A Gaussian process (GP) model regresses displacement information collected over time, and a Markov chain based detector makes use of the direction (heading) information. GP regression is performed together with Median Absolute Deviation to account for contaminated training data. The methodology utilizes the coordinates of a given ferry recorded on a second by second basis via Automatic Identification System. Its effectiveness is demonstrated on a dataset collected in the Solent area.
\end{abstract}

Index Terms - Anomaly Detection, Gaussian Processes, Maritime Traffic, Median Absolute Deviation

\section{INTRODUCTION}

The problem of anomaly detection in machine perception has received substantial interest over the last decade. As the notion of anomaly depends on the context, various systems with different perspectives have been proposed in the literature to address this problem. Conventionally, anomaly is defined as an outlier from some known distribution $[1,2]$ and classical approaches that adhere to this view have been summarized in surveys such as $[3,4,5,6]$.

Among various application domains of anomaly detection, maritime anomaly detection occupies an important place as it aids sea traffic control and collision avoidance, as well as navigation surveillance and detection of illegal marine traffic activity such as piracy, drug smuggling or terrorism. In this study, we concentrate on the special case of detecting anomalous shipping tracks traced by ferries, by using unlabelled data collected via Automated Identification System (AIS).

The compulsory AIS reporting transmitted from ships, over 5000 tonnes, provides information about their positioning in terms of longitude and latitude along with other details like their identification number. It is important to note that although the AIS messages are expected to be transmitted every second, due to the imperfections of the system (such as faulty or incorrectly programmed equipment and the selfmodified, slot based, TDMA on-air access mechanism) and environmental effects impacting range and background noise, the data received will be incomplete.

This work was supported by the Engineering and Physical Sciences Research Council (EPSRC) Grant number EP/K014307/1 and the MOD University Defence Research Collaboration (UDRC) in Signal Processing.
In this paper, we develop an unsupervised learning strategy to automatically label anomalous ferry tracks in a dataset consisting of AIS messages accumulated over a period of time. For this, we make use of feature points such as the ship displacement over time and sailing direction (heading) extracted from the messages to build a set of detectors which are based on Gaussian Processes (for displacement over time) and Markov chains (for direction).

Section 2 discusses the existing techniques in the literature and the relationship of the proposed approach to these methods. Section 3 defines the technical details of our methodology and Section 4 presents the experimental results. Finally, in Section 5, the paper is drawn to conclusion with the discussion of future work.

\section{RELATION TO PRIOR WORK}

In the literature, maritime anomaly detection has been investigated utilizing various methodologies. These include approaches which are based on sets of rules defined by subjectmatter experts $[7,8]$, or algorithms which aim to model normality observed in the given data and use the learned model to carry out anomaly detection by defining a test statistics. The latter are called bottom-up approaches and constitute the majority of the research output in this field.

Grid-based methods, which divide a region of interest into cells to discretize and quantify local information such as speed and placement, have been used in many bottom-up approaches such as $[9,10,11]$. They exploit learning methods such as Hebbian learning, information theory, and Hidden Markov models (HMMs) respectively. Although these methods are popular for their ease of interpretation and applicability, they suffer from low accuracy (especially high false positive rates) and resolution problems for the determination of the grid size.

HMMs are also employed together with other techniques such as clustering to characterize tracks belonging to different ship types in a hierarchical way [12]. Furthermore for the clustering of tracks, studies such as [13] make use of Gaussian Processes (GPs) for the extraction of features, and distance measures like Hellinger distance for their grouping. The main motivation behind using GPs is their flexible and nonparametric nature, avoiding the problems of the estimation of parameters such as the size-of-grid in grid based methods, and the number of Gaussians in Gaussian Mixture Models (GMMs) as used in [14]. 
Besides track modelling, there are various systems concentrating on different aspects of maritime anomaly, such as those focusing on estimating future vessel positions [15, 13], and on global architectures which are directed at detecting anomalies based on multiple sensors [16] or interactions between different learning mechanisms [17].

A thorough review of some of the existing systems and prototypes can be found in [18] where the authors underline the fact that anomaly detection techniques which are also utilized by the maritime problem are already established in the literature, and therefore the main focus of research should now be concentrated on formulating the problems of interest into forms where these techniques can be applied.

In this paper, we consider the problem of anomaly detection in recorded ferry tracks using direction information, time and displacement jointly. As the first set of detectors, GPs are used to model the distance function over the normalized duration of a single trip between two ports. It should be mentioned here that the proposed approach differs from a recent study [19] in terms of exploiting overall trip duration in addition to velocity during regression. Another novelty presented in this study is the use of a data-cleaning system, where the unlabelled training data, which may be corrupted by anomalies, is purified from any outliers by using Median Absolute Deviation (MAD) based on time grids, prior to GP modelling. This facilitates the use of training data without making an unrealistic assumption about the AIS records. The second set of outlier detectors utilizes spatial grids superimposed on the maritime region of interest model direction by employing Markov chains.

\section{METHODOLOGY}

The details of the proposed approach based on the two sets of outlier detectors that utilize Gaussian Processes and Markov chains are presented in Section 3.1 and Section 3.2 respectively. The final combination strategy is then obtained by fusing the decisions of the two classifiers such that if any of the two classifiers detects a track as anomalous, it is taken as anomalous.

\subsection{Displacement Model Based on Gaussian Processes}

In order to define the characteristics of a track, the first feature we exploit is the displacement of a ferry from its departure port, over the total time it takes for the trip. This way, it becomes possible to map the tracks into a unified form, where three critical features: time, displacement and therefore speed are taken into account jointly.

The input data for anomalous ship movement detection is expected to be composed of location information reported in the form $\left[V_{k}, V_{l}\right]$ for a given ship at any time, where $V_{k}$ denotes the longitude and $V_{l}$ the latitude. Given that a ferry operates between the ports $O^{1}$ and $O^{2}$, we initially carry out an automatic extraction of the so-called "one-way trips" between any combination of these ports. Any trip between $O^{1}$ $\& O^{1}$ or $O^{2} \& O^{2}$, where the ferry is coming back to the departure port without visiting the destination is considered to be anomalous, and such trips are left out from the training set, while trips between $O^{1} \& O^{2}$ and $O^{2} \& O^{1}$ are accumulated to be separately processed. Without loss of generality, we can define a ferry's displacement from the departure port $O^{1}$ for a given trip $a$, as a function of the time it spent after the departure:

$$
T_{1}^{a}(\bar{t})=\sqrt{\left(V_{k}^{a}(\bar{t})-O_{k}^{1}\right)^{2}+\left(V_{l}^{a}(\bar{t})-O_{l}^{1}\right)^{2}}
$$

where $O_{k}^{1}$ and $O_{l}^{1}$ are the latitude and longitude coordinates for $O^{1}$ and $\bar{t}$ is the elapsed time. If the duration of the whole journey is given by $t_{l}$, then the displacement over normalized time becomes

$$
f^{a}(s)=f^{a}\left(\frac{\bar{t}}{t_{l}}\right)=T_{1}^{a}(\bar{t})
$$

where $0 \leq s \leq 1$. From Eq. 2 it can observed that for each value of $s, f^{a}(s)$ takes different values depending on $a$. This scenario can be interpreted as having a function $f(s)$ whose value at each $s$ is a random variable.

Gaussian Process regression is a method for stochastically modelling the target value of a variable, by employing a function drawn from a probability distribution [20]. Using $s$ as the variable, the function $f$ can then be denoted as

$$
f(s) \sim G P(b(s), k(s, s))
$$

where $b(s)$ is the mean of the probability distribution at a given realization of $s$ and $k(s, s)$ is the covariance function which represents the similarity between two different realizations, such that

$$
k\left(s_{p}, s_{q}\right)=\operatorname{cov}\left(f\left(s_{p}\right), f\left(s_{q}\right)\right) .
$$

Eq. 4 shows that the covariance between the outputs is given as a function of the inputs. This function is defined in terms of the relative distances of the inputs, with the rationale that the input points which are close to each other are likely to have similar target values [20]. The choice of the covariance matrix should be made according to the requirements of the system. In our approach, we use the squared exponential function, given by

$$
k_{E}\left(s_{p}, s_{q}\right)=\sigma_{f}^{2} \exp \left(-\frac{\left(s_{p}-s_{q}\right)^{2}}{2 h^{2}}\right)
$$

where $h$ is the length-scale specifying the width of the kernel and $\sigma_{f}^{2}$ is the output signal variance which determines the average distance of the function from its mean. Under the assumption of having observations corrupted by independently and identically distributed Gaussian noise with zero mean and $\sigma_{c}^{2}$ variance, the noisy target values can be denoted with variable $\omega$ such that $\omega=f(s)+\epsilon$. This corruption changes the covariance function into

$$
\operatorname{cov}\left(\omega_{p}, \omega_{q}\right)=k\left(s_{p}, s_{q}\right)+\sigma_{c}^{2} \delta_{p, q}
$$

with $\delta$ being the Kronecker delta which is one if and only if $p=q$. 
Let us denote the vector of means belonging to the distributions at each element of the training set, $S$, by $B(S)$; and the matrix obtained after applying the covariance function to all pairs of elements within $S$ as $K(S, S)$. Accordingly, we denote the vector of covariances calculated between a single test point, $s^{*}$, and $S$ as $K\left(S, s^{*}\right)$. Then, given $S$, the distribution informing the prediction at $s^{*}$, namely $f\left(s^{*}\right)$, can be gauged as a Gaussian distribution with the mean and variance

$$
\bar{f}\left(s^{*}\right)=b\left(s^{*}\right)+K\left(S, s^{*}\right)^{T}\left[K(S, S)+\sigma_{c}^{2} I\right]^{-1}(\Omega-B(S))
$$

$\operatorname{var}\left(f\left(s^{*}\right)\right)=k\left(s^{*}, s^{*}\right)-K\left(S, s^{*}\right)^{T}\left[K(S, S)+\sigma_{c}^{2} I\right]^{-1} K\left(S, s^{*}\right)$.

where the vector of target values is denoted by $\Omega$ and $I$ is the identity matrix [20]. The parameters $h, \sigma_{f}^{2}$ and $\sigma_{c}^{2}$ are called the hyperparameters and need to be estimated from the anomaly-free training data. As our training set may be contaminated by anomalies, its initial cleaning has to be carried out before modelling normality, from which deviations will be classified as anomaly.

The cleaning is applied using Median Absolute Deviation from Median (MAD). While dealing with sampled data which is prone to outliers, MAD allows to robustly estimate the standard deviation of the underlying distribution, i.e. it is more resilient to outliers compared to the standard deviation computed from the sampled population space [14]. In our strategy, we discretize the input domain $s \epsilon[0,1]$ into 200 cells, and apply MAD on the target values falling within each cell. MAD is calculated as the median of the absolute differences between the points and their median:

$$
\operatorname{MAD}\left(\Omega_{e}\right)=c \operatorname{med}\left(\operatorname{abs}\left(\Omega_{e}-\operatorname{med}\left(\Omega_{e}\right)\right)\right)
$$

where $\Omega_{e}$ is the vector of target variables in a given cell, $e$, and $c$ is the consistency constant that is equal to 1.4826 when the underlying distribution function is assumed to be Gaussian (as in our case). By setting a cut-off value, $v$, any corrupted data point, $\omega_{e}^{c}$, which satisfies abs $\left(\omega_{e}^{c}-\operatorname{med}\left(\Omega_{e}\right)\right) / \operatorname{MAD}\left(\Omega_{e}\right)>v$ is removed from the training set.

After the application of MAD followed by GP regression, the testing of anomaly for $s^{*}$ is carried out by setting a multiplier $r$ for the desired confidence interval such that

$$
g= \begin{cases}1 & \omega^{*}>\bar{f}\left(s^{*}\right)+r \operatorname{var}\left(f\left(s^{*}\right)\right) \\ 1 & \omega^{*}<\bar{f}\left(s^{*}\right)-r \operatorname{var}\left(f\left(s^{*}\right)\right) \\ 0 & \text { o.w. }\end{cases}
$$

where $g$ is the flag for anomaly, $\omega^{*}$ is the target value for the test sample and $r=2.57$ for $99 \%$ confidence interval. An input trip $a$ is labelled as anomalous if more than $p \%$ of its time stamps are detected as anomalous, where $p$ is a suitable threshold.

\subsection{Sailing Direction Model Based on Markov Chains}

In addition to the displacement of a ferry normalized over time within a single trip, an additional feature we exploit in our approach is the direction of travel at a given location, also known as heading.
Assume that we are analysing a ship's spatial location within a pre-set $n \times m$ grid, $G_{n, m}$. Each cell of this grid can be named as $G_{n, m}(x, y)$ where $x$ is the row index, $y$ is the column index, $1 \leq x \leq n, 1 \leq y \leq m$ and $G_{n, m}(1,1)$ is the left-most cell in the bottom row. For simplicity, we can also refer to each cell as $G_{n, m}(x, y)=G(z)$ where $z=(y-1) m+x$ and $1 \leq x \leq n \times m$. It is then possible to transform an input location $\left[V_{k}, V_{l}\right]$ into the form $G(x, y)$ to find out which cell it falls into, using quantization.

The transition of a ship from a location $G\left(z_{t_{1}}\right)$ at time $t_{1}$ to a location $G\left(z_{t_{2}}\right)$ at a time $t_{2}>t_{1}$ can be modeled as a discrete-time Markov chain, where the likelihood of the ship being in the future state, $G\left(z_{t_{2}}\right)$, depends only on the present state, $G\left(z_{t_{1}}\right)$; and the corresponding transition probability can be denoted by $P\left(z_{t_{2}} \mid z_{t_{1}}\right)$.

We choose the grid cell size dynamically to reflect the distance the ship is able to move in one second's time. By making the assumption that the sailing speed is constant within a localized region, the transition probabilities may then be observed to reflect different directions of travel from a given (present) state. In other words, $P\left(z_{t_{2}} \mid z_{t_{1}}\right)$ can also be denoted as $\bar{P}\left(\theta_{t_{1}} \mid z_{t_{1}}\right)$, where $\theta$ is the variable for the direction parameter from $G\left(z_{t_{1}}\right)$ to $G\left(z_{t_{2}}\right)$ in radians.

In order to accurately estimate the transition probabilities, a large training set with enough data points in each state (cell) is required. As the grid size is defined to be small enough to cover for secondly-moves, in practice, there is only very limited data available for each cell. To be able to compansate for the lack of training data, we propose the use of a second grid layer with a larger cell size fitted onto the original layer.

Let us name the new $k \times l$ grid as $G_{k, l}^{\prime}$; where $k \leq n$ and $l \leq m$; and the corresponding vector form as $G^{\prime}\left(z^{\prime}\right)$. A $G(z)$ cell lying within a given $G^{\prime}\left(z^{\prime}\right)$ can then be indicated by $G(z) \epsilon G^{\prime}\left(z^{\prime}\right)$. The second layer grid cell size is selected so as to cover the first layer cells with similar grid sizes. Doing so, the proposed model aims to come up with a distribution associated to ship behaviour within each cell $G^{\prime}\left(z^{\prime}\right)$, by generalizing over $G(z) \epsilon G^{\prime}\left(z^{\prime}\right)$ through simply marginalizing out the location variable, $z$, such that

$$
\bar{P}_{S\left(z^{\prime}\right)}(\theta)=\sum_{z \in S\left(z^{\prime}\right)} \bar{P}(\theta \mid z) P(G(z))
$$

where $S\left(z^{\prime}\right)$ is the set of $z$ satisfying $G(z) \epsilon G^{\prime}\left(z^{\prime}\right)$ for a given $z^{\prime}$, and $P(G(z))$ is the probability of being in cell $G(z)$. Note that in practice, the calculation in Eq. 10 for a given $z^{\prime}$ can be obtained for all possible $\theta$ using a normalized histogram. After obtaining $\bar{P}_{S\left(z^{\prime}\right)}, \forall z^{\prime}$, an anomaly in a test ship's movement from state $G\left(z_{t_{1}}\right)$ to $G\left(z_{t_{2}}\right)$ within $G^{\prime}\left(z^{\prime}\right)$, can be detected by thresholding the corresponding probability of the obtained direction of travel, $\theta_{t}$. That is,

$$
g= \begin{cases}1 & \bar{P}_{S\left(z^{\prime}\right)}\left(\theta_{t}\right)<r \\ 0 & \text { o.w. }\end{cases}
$$

where $g$ is the flag for anomaly and $r$ is a suitable threshold. As in the case of the GP classifier, a test trip $a$ is labelled as anomalous if more than $p \%$ of its moves related to time stamps are flagged as anomalous. 


\section{EXPERIMENTAL RESULTS}

The experimental study has been conducted on the dataset collected by Thales UK Ltd. at the Solent area between 20/07/2012 and 19/08/2012. The dataset consists of AIS messages received from various vessels occupying the region of interest, out of which ferries have been selected for the application of our methodology. For each ferry, the dataset is split into training and test at a rate of 1:6 respectively, via randomly choosing 4 days for training and 24 for test, and this sampling procedure is repeated.

In order to show the effect of the MAD cleaning, the GP regression obtained for a given training set (based on trips from one of the ports) is depicted in Fig. 1 where the training samples after cleaning are represented by dark dots (red), and those cleaned during MAD are represented by light colour (green). In our experiments, by setting the confidence constant $c=1.4826,3.4 \%$ of the training data is detected as anomalous/corrupted. The mean of the GP regression, which is then fitted onto the purified data is provided by light circles (yellow), and the 95\% confidence intervals are depicted by dark circles (black).

As for the necessity of the MC classifier based on direction information to be used in addition to GPs, in Fig. 2 we show two anomalous tracks which are identified by this classifier but cannot be detected by the GP based detector. The track indicated by the dashed lines, where the ferry is performing a sudden sharp turn is taken from the test set, whereas the track indicated by the solid line is artificially created.

In Fig. 3, we carry out an assessment of the tracks that are classified as anomalous together with those that are labelled normal by the algorithm. For an example test set, the tracks labelled anomalous are provided in dark shade (red) whereas the normal tracks are given in lighter colour (yellow). Using a $90 \%$ confidence interval during the GP regression, a threshold of $r=0.0005$ for the MC direction classifier, and an anomaly threshold of $p=30$ for both classifiers, we obtain a $93 \%$ detection rate for anomalies, and $2 \%$ false positive rate (FPR). It is also possible to detect all anomalies with FPR $=6 \%$. The ROC curve using confidence intervals $[1,0.99,0.95,0.90,0.85,0.80,0.75,0.50]$ is given in Fig. 4.

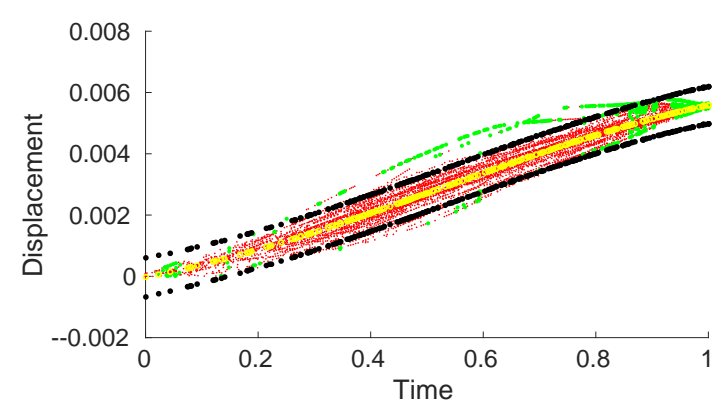

Fig. 1. GP regression on training data after MAD cleaning

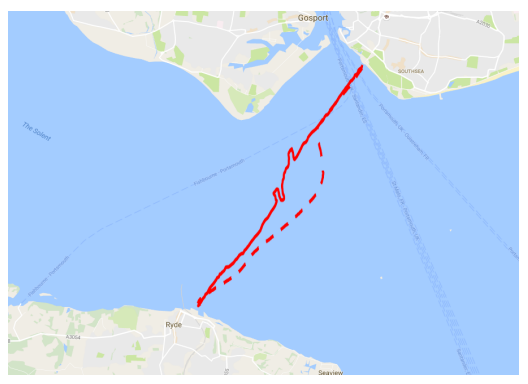

Fig. 2. Anomalous tracks identified by the MC classifier which cannot be detected by the GP classifier

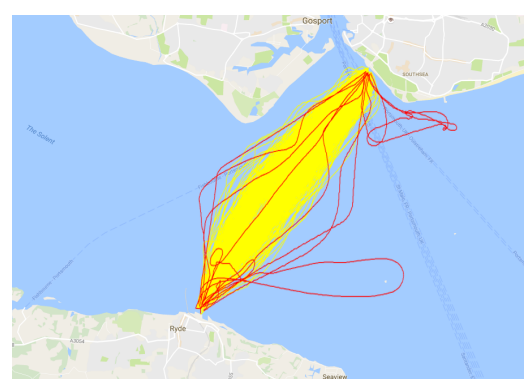

Fig. 3. Detected anomalies plotted on top of normalities (dark shade on light)

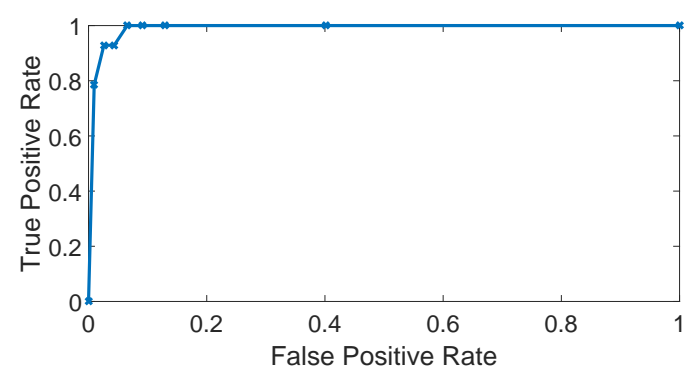

Fig. 4. ROC analysis for the proposed methodology

\section{CONCLUSIONS}

In this paper, we present a novel approach for detecting anomalous tracks in an unlabelled dataset, by using features involving speed, time and direction information. Gaussian Processes and Markov chains have been utilized for this purpose, together with the application of Medium Absolute Deviation for the initial cleaning of the training data before the application of GPs. The method is shown to successfully detect all anomalies in an example dataset with a FPR of 6\%, although a more detailed experimental study involving other datasets is left for future work. Moreover, the application of MAD on the MC classifier can also be addressed to see its effect on the possible reduction of the false positive rate. 


\section{REFERENCES}

[1] D. Agarwal, "Detecting anomalies in cross-classified streams: A bayesian approach," Knowl. Inf. Syst., vol. 11, no. 1, pp. 29-44, Dec. 2006.

[2] F. J. Anscombe and I. Guttman, "Rejection of outliers," Technometrics, vol. 2:2, pp. 123-147, 1960.

[3] M. Markou and S. Singh, "Novelty detection: A reviewpart 1: Statistical approaches," Signal Processing, vol. 83:12, pp. 2481-2497, 2003.

[4] M. Markou and S. Singh, "Novelty detection: A reviewpart 2: Neural network based approaches," Signal Processing, vol. 83:12, pp. 2499-2521, 2003.

[5] V. Hodge and J. Austin, "A survey of outlier detection methodologies," Artif. Intell. Rev., vol. 22, no. 2, pp. 85-126, Oct. 2004.

[6] M. Agyemang, K. Barker, and R. Alhajj, "A comprehensive survey of numeric and symbolic outlier mining techniques," Intelligent Data Analysis, vol. 10:6, pp. 521-538, 2006.

[7] R. Jasinevicius and V. Petrauskas, "Fuzzy expert maps for risk management systems," in 2008 IEEE/OES US/EU-Baltic International Symposium, May 2008, pp. $1-4$.

[8] J. Roy and M. Davenport, "Exploitation of maritime domain ontologies for anomaly detection and threat analysis," in 2010 International WaterSide Security Conference, Nov 2010, pp. 1-8.

[9] N. A. Bomberger, B. J. Rhodes, M. Seibert, and A. M. Waxman, "Associative learning of vessel motion patterns for maritime situation awareness," in 2006 9th International Conference on Information Fusion, July 2006, pp. 1-8.

[10] D. Barbara, "An information theoretic appriach to anomaly detection," in Workshop on Detection of Anomalous Behaviors in Maritime Environments, 2009.

[11] C. Griffin, "Learning and prediction for readiness: An onr office 31 program," in TTCP MAR AG, 2009.

[12] G. K. D. de Vries, V. Malaise, M. van Someren, P. Adriaans, and G. Schreiber, "Semi-automatic ontology extension in the maritime domain," in Proc. of the 20th Belgian-Netherlands Conference on Artificial Intelligence (BNAIC 2008), 2008.

[13] M. Smith, S. Reeche, S. Roberts, I. Psorakis, and I. Rezek, "Maritime abnormality detection using gaussian processes," Knowledge and Information Systems, vol. 38, no. 3, pp. 717-741, 2014.
[14] C. Leys, C. Ley, O. Klein, P. Bernard, and L. Licata, "Detecting outliers: Do not use standard deviation around the mean, use absolute deviation around the median," Journal of Experimental Social Psychology, vol. 49, no. 4, pp. $764-766,2013$.

[15] B. Ristic, B. La Scala, M. Morelande, and N. Gordon, "Statistical analysis of motion patterns in ais data: Anomaly detection and motion prediction," in Information Fusion, 2008 11th International Conference on, June 2008, pp. 1-7.

[16] M. Seibert, "Maritime anomaly detection," in Workshop on Detection of Anomalous Behaviors in Maritime Environments, 2009.

[17] J. Tozicka, M. Rovatsos, M. Pechoucek, and S. Urban, "Malef\&\#58; framework for distributed machine learning and data mining," Int. J. Intell. Inf. Database Syst., vol. 2, no. 1, pp. 6-24, Feb. 2008.

[18] E. Martineau and J. Roy, Maritime Anomaly Detection: Domain Introduction and Review of Selected Literature, Technical memorandum. 2011.

[19] K. Kowalska and L. Peel, "Maritime anomaly detection using gaussian process active learning," in Information Fusion (FUSION), 2012 15th International Conference on, July 2012, pp. 1164-1171.

[20] C. E. Rasmussen and C. K. I. Williams, Gaussian Processes for Machine Learning (Adaptive Computation and Machine Learning), The MIT Press, 2005. 